ANNALES

POLONICI MATHEMATICI

$90.2(2007)$

\title{
Hodge type decomposition
}

\author{
by WojcIEch KOzŁowski (Łódź)
}

\begin{abstract}
In the space $\Lambda^{p}$ of polynomial $p$-forms in $\mathbb{R}^{n}$ we introduce some special inner product. Let $\mathbf{H}^{p}$ be the space of polynomial $p$-forms which are both closed and co-closed. We prove in a purely algebraic way that $\Lambda^{p}$ splits as the direct sum $d^{\star}\left(\Lambda^{p+1}\right) \oplus$ $\delta^{\star}\left(\Lambda^{p-1}\right) \oplus \mathbf{H}^{p}$, where $d^{\star}\left(\operatorname{resp} . \delta^{\star}\right)$ denotes the adjoint operator to $d$ (resp. $\delta$ ) with respect to that inner product.
\end{abstract}

1. Introduction and main result. To begin with, recall the classical Hodge decomposition theorem. Suppose $\left(M^{n}, g\right)$ is an oriented Riemannian manifold. Introduce the following notation: $\Lambda^{p}(M)$ is the space of all smooth differential $p$-forms on $M$ whereas $d, \delta$ and $\Delta=d \delta+\delta d$, are the differential, co-differential and the Laplace-Beltrami operator, respectively. Recall that for any $\omega \in \Lambda^{p}(M), \delta \omega=(-1)^{n(p+1)+1} \star d \star \omega$, where $\star$ is the Hodge operator. Moreover, let $\mathbf{H}^{p}(M)$ denote the space of $p$-forms which are both closed and co-closed: $\mathbf{H}^{p}(M)=\left\{\omega \in \Lambda^{p}(M): d \omega=\delta \omega=0\right\}$.

If $M$ is compact then the formula

$$
(\omega \mid \eta)_{M}=\int_{M} \omega \wedge \star \eta
$$

defines an inner product in $\Lambda^{p}(M)$. Then $d^{\star}=\delta$ and $\delta^{\star}=d$, i.e., $d$ and $\delta$ are adjoint to each other. Take any $\omega \in \Lambda^{p}(M)$; then $(\omega \mid \Delta \omega)_{M}=(d \omega \mid d \omega)_{M}+$ $(\delta \omega \mid \delta \omega)_{M}$. Therefore, $\omega$ is harmonic, i.e., $\Delta \omega=0$, iff $\omega \in \mathbf{H}^{p}(M)$. The following is a classical result in analysis:

TheOREM 1.1 (Hodge decomposition theorem). On a compact oriented Riemannian manifold $M, \Lambda^{p}(M)=d \Lambda^{p-1}(M) \oplus^{\perp} \delta \Lambda^{p+1}(M) \oplus^{\perp} \mathbf{H}^{p}(M)$, or equivalently $\Lambda^{p}(M)=\delta^{\star} \Lambda^{p-1}(M) \oplus^{\perp} d^{\star} \Lambda^{p+1}(M) \oplus^{\perp} \mathbf{H}^{p}(M)$, where $\oplus^{\perp}$ denotes an orthogonal direct sum.

For the proof of the Hodge decomposition theorem we refer to the book of F. Warner ([4, Chapter 6]). Notice that a historical survey of the devel-

2000 Mathematics Subject Classification: 33C55, 35J99, 53C43.

Key words and phrases: Hodge theorem, polynomial $p$-form. 
opment of the theory of elliptic operators is given in the beautiful article of L. Hörmander ([1]).

We treat $\mathbb{R}^{n}$ as a Riemannian manifold equipped with the canonical inner product. If $f$ is a polynomial of the form $f=\sum_{\alpha} a_{\alpha} x^{\alpha}$ (here $\alpha=$ $\left(\alpha_{1}, \ldots, \alpha_{n}\right)$ denotes a multi-index $)$ we put

$$
f(D)=\sum_{\alpha} a_{\alpha} D^{\alpha}, \quad D^{\alpha}=\frac{\partial^{\alpha_{1}+\cdots+\alpha_{n}}}{\left(\partial x^{1}\right)^{\alpha_{1}} \cdots\left(\partial x^{n}\right)^{\alpha_{n}}} .
$$

Let $\mathcal{P}_{k}$ (resp. $\mathcal{H}_{k}$ ) denote the space of all homogeneous (resp. harmonic homogeneous) polynomials on $\mathbb{R}^{n}$ of degree $k$. Define the inner product $(\cdot, \cdot)=(\cdot, \cdot)_{k}$ in $\mathcal{P}_{k}$ as follows: $(f, g)=f(D) g$, for $f, g \in \mathcal{P}_{k}$ (cf. [3, p. 139]). Clearly, for any $f \in \mathcal{P}_{k}, g \in \mathcal{P}_{l}$ and $h \in \mathcal{P}_{k+l},(g f, h)_{k+l}=(f, g(D) h)_{k}$. In particular $\left(^{1}\right),(\Delta f, h)=\left(f,-r^{2} h\right)$ where $r^{2}(x)$ is the square of the Euclidian norm of $x \in \mathbb{R}^{n}$. As a result we get the well-known identity ([3, Thm. 2.12])

$$
\mathcal{P}_{k}=\mathcal{H}_{k} \oplus^{\perp} r^{2} \mathcal{P}_{k-2} .
$$

We may extend (using linearity) this inner product onto the space of all polynomials, putting $(f, g)=0$ if $f \in \mathcal{P}_{k}, g \in \mathcal{P}_{l}$ and $k \neq l$.

Recall that any $p$-form $\omega$ in $\mathbb{R}^{n}$ has a unique expression

$$
\omega=\frac{1}{p !} \sum_{i_{1}, \ldots, i_{p}=1}^{n} \omega_{i_{1}, \ldots, i_{p}} d x^{i_{1}} \wedge \cdots \wedge d x^{i_{p}}
$$

where the functions $\omega_{i_{1}, \ldots, i_{p}}$, called coefficients, are skew-symmetric with respect to the indices. Denote by $\Lambda^{p}$ the space of all $p$-forms in $\mathbb{R}^{n}$ that have polynomial coefficients. We put $\Lambda^{p}=\{0\}$ if $p<0$. Let $\mathfrak{H}^{p}$ and $\mathbf{H}^{p}$ denote, respectively, the space of all harmonic polynomial $p$-forms and its subspace of all polynomial $p$-forms which are both closed and co-closed:

$$
\mathfrak{H}^{p}=\left\{\omega \in \Lambda^{p}: \Delta \omega=(d \delta+\delta d) \omega=0\right\}, \quad \mathbf{H}^{p}=\left\{\omega \in \Lambda^{p}: d \omega=\delta \omega=0\right\} .
$$

Consider the vector field $\nu$ and the 1 -form $\nu^{\star}$ defined by

$$
\nu_{x}=x^{1} \frac{\partial}{\partial x^{1}}+\cdots+x^{n} \frac{\partial}{\partial x^{n}}, \quad \nu_{x}^{\star}=x^{1} d x^{1}+\cdots+x^{n} d x^{n},
$$

where $x=\left(x^{1}, \ldots, x^{n}\right) \in \mathbb{R}^{n}$. One sees that $\nu^{\star} \nu=r^{2}$. Let $\varepsilon_{\nu}\left(\right.$ resp. $\left.\iota_{\nu}\right)$ denote the exterior (resp. interior) product, i.e., $\varepsilon_{\nu} \omega=\nu^{\star} \wedge \omega$ and $\iota_{\nu} \omega=$ $\omega(\nu, \cdot, \ldots, \cdot)$. Manifestly, $\varepsilon_{\nu}^{2}=0$ and $\iota_{\nu}^{2}=0$. Since $\iota_{\nu}$ is an anti-derivation, $\iota_{\nu} \varepsilon_{\nu} \omega=\nu^{\star} \nu \omega-\varepsilon_{\nu} \iota_{\nu} \omega$. Therefore, we get

$$
\left(\iota_{\nu} \varepsilon_{\nu}+\varepsilon_{\nu} \iota_{\nu}\right) \omega=r^{2} \omega
$$

One can easily check that

$$
d \varepsilon_{\nu}=-\varepsilon_{\nu} d, \quad \delta \iota_{\nu}=-\iota_{\nu} \delta .
$$

$\left({ }^{1}\right)$ Since we define the Laplace operator as $\Delta=d \delta+\delta d$, our $\Delta$ on smooth real-valued functions has the sign such that $\Delta u=-\left(\partial /\left(\partial x^{1}\right)^{2}+\cdots+\partial /\left(\partial x^{n}\right)^{2}\right) u$ on $\mathbb{R}^{n}$. 
Equip the space $\Lambda^{p}$ with the inner product $(\cdot \mid \cdot)$ as follows: for any $\omega, \eta \in$ $\Lambda^{p}$ we put

$$
(\omega \mid \eta)=\frac{1}{p !} \sum_{i_{1}, \ldots, i_{p}=1}^{n}\left(\omega_{i_{1}, \ldots, i_{p}}, \eta_{i_{1}, \ldots, i_{p}}\right),
$$

where $\omega_{i_{1}, \ldots, i_{p}}$ 's and $\eta_{i_{1}, \ldots, i_{p}}$ 's denote the coefficients of $\omega$ and $\eta$, respectively.

It turns out ([2, Thm. 2.2.1]) that the operators $\delta$ and $-\varepsilon_{\nu}$, and $d$ and $\iota_{\nu}$, are adjoint to each other:

$$
\delta^{\star}=-\varepsilon_{\nu}, \quad d^{\star}=\iota_{\nu} .
$$

If $\omega$ is a polynomial form such that $\iota_{\nu} \omega=\varepsilon_{\nu} \omega=0$ then, by (1.2), $\omega=0$. Since $\iota_{\nu}^{2}=\varepsilon_{\nu}^{2}=0$, we see that $\varepsilon_{\nu}\left(\Lambda^{p-1}\right) \cap \iota_{\nu}\left(\Lambda^{p+1}\right)=\{0\}$. Moreover, by (1.4) the spaces $\mathbf{H}^{p}$ and $\varepsilon_{\nu}\left(\Lambda^{p-1}\right) \oplus \iota_{\nu}\left(\Lambda^{p+1}\right)$ are mutually orthogonal.

The purpose of this paper is to prove, in a purely algebraic way, the following

Theorem 1.2 (Hodge type decomposition). For any $0 \leq p \leq n$, the space $\Lambda^{p}$ splits as the direct sum $\Lambda^{p}=\iota_{\nu} \Lambda^{p+1} \oplus \varepsilon_{\nu} \Lambda^{p-1} \oplus \mathbf{H}^{p}$, or equivalently $\Lambda^{p}=d^{\star} \Lambda^{p+1} \oplus \delta^{\star} \Lambda^{p-1} \oplus \mathbf{H}^{p}$.

2. Some preparations. Denote by $\Lambda_{k}^{p}$ and $\mathfrak{H}_{k}^{p}$ the space of all forms in $\mathbb{R}^{n}$ that have coefficients from $\mathcal{P}_{k}$ and $\mathcal{H}_{k}$, respectively. It is convenient to put $\Lambda_{k}^{p}=\{0\}$ if either $p<0$ or $k<0$. Let $\mathbf{H}_{k}^{p}=\mathbf{H}^{p} \cap \Lambda_{k}^{p}=\left\{\omega \in \Lambda_{k}^{p}: d \omega=\right.$ $\delta \omega=0\}$. Since for any differential $p$-form $\omega$ on $\mathbb{R}^{n}$,

$$
(\Delta \omega)_{i_{1}, \ldots, i_{p}}=\Delta \omega_{i_{1}, \ldots, i_{p}},
$$

we see that $\omega \in \mathfrak{H}^{p}$ iff all coefficients of $\omega$ are harmonic polynomials. In particular, $\mathfrak{H}_{k}^{p}=\mathfrak{H}^{p} \cap \Lambda_{k}^{p}$ and $\mathbf{H}_{k}^{p}=\mathfrak{H}_{k}^{p} \cap \mathbf{H}^{p}$. Moreover, the decomposition (1.1) translates immediately to $\Lambda_{k}^{p}$ where we have

$$
\Lambda_{k}^{p}=\mathfrak{H}_{k}^{p} \oplus^{\perp} r^{2} \Lambda_{k-2}^{p} .
$$

In the proof of the main theorem we will need to use some formulæ from [2]. We list them below.

For any $\omega \in \Lambda_{k}^{p}$ we have ([2, Prop. 2.2.1])

$$
\begin{aligned}
\delta \varepsilon_{\nu} \omega & =-\varepsilon_{\nu} \delta \omega-(n-p+k) \omega, \\
d \iota_{\nu} \omega & =-\iota_{\nu} d \omega+(p+k) \omega .
\end{aligned}
$$

Applying the second identity in (1.3) and (2.3) we see that for any polynomial form $\omega$,

$$
\Delta \iota_{\nu} \omega=\iota_{\nu} \Delta \omega+2 \delta \omega
$$


Define the spaces $\chi_{p, k}, \chi_{p, k}^{0}$ and the operator $I_{p . k}$ as follows:

$$
\chi_{p, k}=\mathfrak{H}_{k}^{p} \cap \operatorname{ker} \delta, \quad \chi_{p, k}^{0}=\chi_{p, k} \cap \operatorname{ker} \iota_{\nu},
$$

$I_{p . k}=\varepsilon_{\nu}-c_{k} r^{2} d, \quad$ where $\quad c_{k}= \begin{cases}1 /(n+2 k-4) & \text { if } k \geq 2,0<p \leq n, \\ 0 & \text { otherwise }\end{cases}$

We have the following decompositions ([2, (3.3.2), (3.3.3)]):

$$
\begin{gathered}
\chi_{p, k}=\chi_{p, k}^{0} \oplus^{\perp} d \chi_{p-1, k+1}^{0}, \\
\mathfrak{H}_{k}^{p}=\chi_{p, k}^{0} \oplus^{\perp} d \chi_{p-1, k+1}^{0} \oplus^{\perp} \varepsilon_{\nu} d \chi_{p-2, k}^{0} \oplus^{\perp} I_{p, k}\left(\chi_{p-1, k-1}^{0}\right) .
\end{gathered}
$$

Notice that in (2.6) some subspaces may degenerate sometimes. In particular, by $(2.3)$ it follows easily $([2,(3.2 .1)])$ that

$$
\chi_{p, 0}^{0}=\{0\} \quad \text { if } p>0 .
$$

REMARK. The orthogonal decomposition (2.6) above is the key step in the proof of Hodge type decomposition. In fact, (2.6) is the very special case of the more general decomposition [2, Thm. 3.3.1] of the kernel of the operator $L=a d \delta+b \delta d, a, b>0$.

The last part of this section has a technical character. From (1.3) and (2.4) it follows that $\iota_{\nu} \mathbf{H}_{k-1}^{p+1} \subset \chi_{p, k}^{0}$. On the other hand, if $p+k>0$ then taking $\omega \in \chi_{p, k}^{0}$ we see that $\omega=\iota_{\nu} \eta$, where $\eta=(p+k)^{-1} d \omega$. Clearly, $\eta \in \mathbf{H}_{k-1}^{p+1}$. So we have

$$
\iota_{\nu} \mathbf{H}_{k-1}^{p+1}=\chi_{p, k}^{0} \quad \text { if } p+k>0 .
$$

If $k=p=0$ then clearly $\mathbf{H}_{0}^{0}=\mathbb{R}$ (the space of constant functions). Suppose that either $p>0$ or $k>0$. Let $\omega \in \mathbf{H}_{k}^{p}$. In the light of (2.5) and the relation $\mathbf{H}_{k}^{p} \subset \chi_{p, k}$ we may write $\omega=\omega^{\prime}+\omega^{\prime \prime}$, where $\omega^{\prime} \in \chi_{p, k}^{0}$ and $\omega^{\prime \prime} \in d \chi_{p-1, k+1}$. Since $\omega$ and $\omega^{\prime \prime}$ are closed, $d \omega^{\prime}=0$. Therefore, $0=\iota_{\nu} d \omega^{\prime}=$ $-d \iota_{\nu} \omega^{\prime}+(p+k) \omega^{\prime}=(p+k) \omega^{\prime}$. Since $p+k>0, \omega^{\prime}=0$. Thus $\mathbf{H}_{k}^{p} \subset d \chi_{p-1, k+1}^{0}$. On the other hand, one easily checks that $d \chi_{p-1, k+1}^{0} \subset \mathbf{H}_{k}^{p}$. We have proved that

$$
\mathbf{H}_{k}^{p}= \begin{cases}\mathbb{R} & \text { if } p=k=0, \\ d \chi_{p-1, k+1}^{0} & \text { otherwise. }\end{cases}
$$

Let us complete the section with the following observation: if either $p \neq 1$ or $k \neq 1$ then

$$
I_{p, k}\left(\chi_{p-1, k-1}^{0}\right) \oplus r^{2} \Lambda_{k-2}^{p} \subset \iota_{\nu} \varepsilon_{\nu}\left(\Lambda_{k-2}^{p}\right) \oplus \varepsilon_{\nu} \iota_{\nu}\left(\Lambda_{k-2}^{p}\right) .
$$

Indeed, the inclusion $r^{2} \Lambda_{k-2}^{p} \subset \iota_{\nu} \varepsilon_{\nu}\left(\Lambda_{k-2}^{p}\right) \oplus \varepsilon_{\nu} \iota_{\nu}\left(\Lambda_{k-2}^{p}\right)$ follows from (1.2). Now we prove that $I_{p, k}\left(\chi_{p-1, k-1}^{0}\right) \subset \iota_{\nu} \varepsilon_{\nu}\left(\Lambda_{k-2}^{p}\right) \oplus \varepsilon_{\nu} \iota_{\nu}\left(\Lambda_{k-2}^{p}\right)$. If $p=0$ or $k=0$ then the inclusion is trivial. Suppose that $p, k>0$. Take $\omega \in$ 
$I_{p, k}\left(\chi_{p-1, k-1}^{0}\right), \omega=I_{p, k} \eta, \eta \in \chi_{p-1, k-1}^{0}$. Using (1.2) and (2.3) one easily checks that $\omega=\iota_{\nu} \varepsilon_{\nu} \psi^{\prime}+\varepsilon_{\nu} \iota_{\nu} \psi^{\prime \prime}$, where

$$
\psi^{\prime}=-c_{k} d \eta, \quad \psi^{\prime \prime}=\frac{1-(p+k-2) c_{k}}{p+k-2} d \eta
$$

3. Proof of the Hodge type decomposition. To prove Theorem 1.2 it suffices to show that for any $p, k \geq 0$,

$$
\Lambda_{k}^{p}=\iota_{\nu} \Lambda_{k-1}^{p+1} \oplus \varepsilon_{\nu} \Lambda_{k-1}^{p-1} \oplus \mathbf{H}_{k}^{p} .
$$

To prove (3.1) we apply induction with respect to $k$. Before doing this, we check some very special case of (3.1) separately. Namely, we have

$$
\Lambda_{1}^{1}=\iota_{\nu} \Lambda_{0}^{2} \oplus \varepsilon_{\nu} \Lambda_{0}^{0} \oplus \mathbf{H}_{1}^{1} .
$$

Proof of (3.2). Clearly, $\Lambda_{1}^{1}=\mathfrak{H}_{1}^{1}, \Lambda_{0}^{0}=\chi_{0,0}^{0}=\mathbb{R}$ and $\Lambda_{0}^{2}=\mathfrak{H}_{0}^{2}$. Formula (2.6) implies that it suffices to show that $\chi_{1,1}^{0}=\iota_{\nu} \mathfrak{H}_{0}^{2}$. By $(1.3), \iota_{\nu} \mathfrak{H}_{0}^{2} \subset \chi_{1,1}^{0}$. Let $\omega \in \chi_{1,1}^{0}$. Put $\eta=(1 / 2) d \omega$; then one can easily check that $\iota_{\nu} \eta=\omega$. Thus $\chi_{1,1}^{0}=\iota_{\nu} \mathfrak{H}_{0}^{p}$.

Proof of (3.1) by induction with respect to $k$. Suppose that $k=0$. If $p=0$ then (3.1) is a direct consequence of the equalities $\Lambda_{0}^{0}=\mathfrak{H}_{0}^{0}=\mathbf{H}_{0}^{0}=\mathbb{R}$. If $p>0$ then (3.1) follows from (2.9), (2.6), (2.7) and the equality $\Lambda_{0}^{p}=\mathfrak{H}_{0}^{p}$.

Suppose that (3.1) holds for $k-1, k \geq 1$. Take any $p \geq 0$. We may assume that either $p \neq 1$ or $k \neq 1$ (see (3.2)). Using (1.4), (2.2) and (2.3) we find that $\iota_{\nu} \varepsilon_{\nu}\left(\Lambda_{k-2}^{p}\right)$ and $\iota_{\nu}\left(\mathbf{H}_{k-1}^{p+1}\right)$, and $\varepsilon_{\nu} \iota_{\nu}\left(\Lambda_{k-2}^{p}\right)$ and $\varepsilon_{\nu}\left(\mathbf{H}_{k-1}^{p-1}\right)$ are mutually orthogonal. Thus, by induction hypothesis we have

$$
\begin{aligned}
& \varepsilon_{\nu}\left(\Lambda_{k-1}^{p-1}\right)=\varepsilon_{\nu} \iota_{\nu}\left(\Lambda_{k-2}^{p}\right) \oplus \varepsilon_{\nu}\left(\mathbf{H}_{k-1}^{p-1}\right), \\
& \iota_{\nu}\left(\Lambda_{k-1}^{p+1}\right)=\iota_{\nu} \varepsilon_{\nu}\left(\Lambda_{k-2}^{p}\right) \oplus \iota_{\nu}\left(\mathbf{H}_{k-1}^{p+1}\right) .
\end{aligned}
$$

From (2.1), (2.6), (2.8), (2.9), (2.10) and (3.3) we get

$$
\begin{array}{rll}
\Lambda_{k}^{p} & \stackrel{(\supset .3)}{\supset} & \varepsilon_{\nu}\left(\Lambda_{k-1}^{p-1}\right) \oplus \iota_{\nu}\left(\Lambda_{k-1}^{p+1}\right) \oplus \mathbf{H}_{k}^{p} \\
& \stackrel{(3.3)}{=} & \varepsilon_{\nu} \iota_{\nu}\left(\Lambda_{k-2}^{p}\right) \oplus \iota_{\nu} \varepsilon_{\nu}\left(\Lambda_{k-2}^{p}\right) \oplus \varepsilon_{\nu}\left(\mathbf{H}_{k-1}^{p-1}\right) \oplus \iota_{\nu}\left(\mathbf{H}_{k-1}^{p+1}\right) \oplus \mathbf{H}_{k}^{p} \\
& \stackrel{(2.9),(2.8)}{=} & \varepsilon_{\nu} \iota_{\nu}\left(\Lambda_{k-2}^{p}\right) \oplus \iota_{\nu} \varepsilon_{\nu}\left(\Lambda_{k-2}^{p}\right) \oplus \varepsilon_{\nu} d \chi_{p-2, k} \oplus \chi_{p, k}^{0} \oplus \mathbf{H}_{k}^{p} \\
& \stackrel{(2.10)}{\supset} & r^{2} \Lambda_{k-2}^{p} \oplus I_{p, k}\left(\chi_{p-1, k-1}^{0}\right) \oplus \chi_{p, k}^{0} \oplus \varepsilon_{\nu} d \chi_{p-2, k} \oplus \mathbf{H}_{k}^{p} \\
& \stackrel{(2.6)}{=} & r^{2} \Lambda_{k-2}^{p} \oplus \mathfrak{H}_{k}^{p} \\
& \stackrel{(2.1)}{=} & \Lambda_{k}^{p},
\end{array}
$$

which proves (3.1).

Induction completes the proof. 


\section{References}

[1] L. Hörmander, A history of existence theorems for the Cauchy-Riemann complex in $L^{2}$-space, J. Geom. Anal. 13 (2003), 329-357.

[2] W. Kozłowski, Laplace type operators: Dirichlet problem, Ann. Scoula Norm. Sup. Pisa, to appear.

[3] E. M. Stein and G. Weiss, Fourier Analysis on Euclidean Spaces, Princeton Univ. Press, 1971.

[4] F. W. Warner, Foundations of Differentiable Manifolds and Lie Groups, Scott \& Foresman, London, 1971.

Institute of Mathematics

Polish Academy of Sciences

Łódź Branch
Faculty of Mathematics

Łódź University

Banacha 22

90-238 Łódź, Poland

E-mail:wojciech@math.uni.lodz.pl

Received 10.10.2005

and in final form 15.12.2006 